\title{
Enhancing clinical trial development for pediatric kidney diseases
}

\author{
H. William Schnaper ${ }^{1}$, Joseph T. Flynn ${ }^{2}$, Coleman Gross ${ }^{3}$, Anne B. Cropp ${ }^{4}$, Bastian Dehmel ${ }^{5}$, Leah B. Patel ${ }^{6}$, \\ Larry A. Greenbaum ${ }^{7}$, Elisabeth Houtsmuller ${ }^{8}$, Frederick Kaskel ${ }^{9}$, Marva Moxey-Mims ${ }^{10}$, Karen Nowak ${ }^{6}$, Douglas Silverstein ${ }^{11}$, \\ Aliza Thompson ${ }^{12}$, Lynne Yao ${ }^{13}$, Edress Darsey ${ }^{14}$ and William E. Smoyer ${ }^{15}$
}

The conduct of clinical trials in small pediatric subspecialties such as pediatric nephrology is hampered by both clinical demands on the pediatric nephrologist and the small number of appropriate patients available for such studies. The American Society of Pediatric Nephrology Therapeutics Development Committee (TDC) was established to (1) identify the various stakeholders with interests and/or expertise related to clinical trials in children with kidney disease and (2) develop more effective partnerships among all parties regarding strategies for successful clinical trial development and execution. This article discusses the rationale, structure, and function of the TDC, the status of progress toward its goals, and the insights gained to date that may be useful for other subspecialties that face similar challenges.

C onsiderable attention has been directed recently toward the limited availability of effective, U.S. Food and Drug Administration (FDA)-approved drugs for children in the United States. (1) It has been estimated that only $46 \%$ of FDA-approved products include labeling information for pediatric use $(2,3)$ and the percentage may be even lower for younger children. Indeed, for premature infants, it approaches zero. Although some of the treatments used "off label" for the pediatric population have a clear empirical value, scientific validation of efficacy and safety remains unconfirmed for many. To address this issue, a series of legislative initiatives have created incentives and requirements for manufacturers to conduct studies in children. The Best Pharmaceuticals for Children Act (BPCA; ref: Public Law 107-109, 115 Stat. 1408 (4 January 2002) offers pharmaceutical companies an additional 6 months of marketing exclusivity for drugs that have been studied in a pediatric population. This is believed to confer a public health benefit for children, but BPCA does not require that the trial is positive or shows statistically significant efficacy. The Pediatric Research Equity Act (PREA: ref: Public Law 108-155, 117 Stat. 1936 (3 December 2003)) requires manufacturers to conduct studies in children under certain conditions. $(4,5)$ Similar legislation is in effect in the European Union. $(6,7)$ Parallel to the situation with drugs, few devices have been approved or cleared for use in children. At this time, manufacturers are not required to study children during device development. As a result, off-label use of medical devices in children is relatively common. In the absence of specific legislation such as BPCA and PREA for devices, the Center for Devices and Radiological Health (CDRH) at the FDA has explored strategies to enhance the inclusion of children in device studies. One product of those efforts was the release of a guidance document (8) in June 2016. This guidance proposes to use data obtained from adult study participants or from pediatric populations treated for other conditions to provide information to assist the physician in making a more informed decision.

Although off-label use is considered appropriate when exercised in the best judgment of a practitioner, (9) specific determination of safety and efficacy would be ideal. However, there are significant impediments to conducting drug and device studies in children. Market incentives for developing pediatric applications are generally minimal. In addition to high initial cost and low potential return on investment for biopharmaceutical and device companies, the number of trial participants available for pediatric studies is typically less. Pediatric diseases tend to be more heterogeneous in cause than adult diseases, and a small number of potential pediatric trial participants are scattered across disparate programs throughout the United States and abroad. Financial pressures

\footnotetext{
${ }^{1}$ Department of Pediatrics (Kidney Diseases), Northwestern University Feinberg School of Medicine, Chicago, Illinois; ${ }^{2}$ Division of Nephrology, Department of Pediatrics, Seattle Children's Hospital, Seattle, Washington; ${ }^{3}$ Relypsa, Inc., a Vifor Pharma Company, Redwood City, California; ${ }^{4}$ Echelon Pharma Solutions LLC, Madison, Connecticut; ${ }^{5}$ Amgen Europe $\mathrm{GmbH}$, Zug, Switzerland; ${ }^{6}$ Mallinckrodt Pharmaceuticals, Bedminster, New Jersey; ${ }^{7}$ Department of Pediatrics, Children's Healthcare of Atlanta and Emory University, Atlanta, Georgia; ${ }^{8}$ Science, Healthcare Delivery and Disparities Research, PCORI, Washington, DC; ${ }^{9}$ Department of Pediatrics, Montefiore Medical Center, Bronx, New York; ${ }^{10}$ National Institute of Diabetes, and Digestive, and Kidney Diseases, National Institutes of Health, Bethesda, Maryland; ${ }^{11}$ Center for Devices and Radiological Health, Renal Devices Branch, U.S. Food and Drug Administration, Silver Spring, Maryland; ${ }^{12}$ Center for Drug Evaluation and Research, Division of Cardiovascular and Renal Products, U.S. Food and Drug Administration, Silver Spring, Maryland; ${ }^{13}$ Center for Drug Evaluation and Research, Division of Pediatrics and Maternal Health, U.S. Food and Drug Administration, Silver Spring, Maryland; ${ }^{14}$ Pfizer Corporation, New York City, New York; ${ }^{15}$ Center for Clinical and Translational Research, The Research Institute at Nationwide Children's Hospital, Columbus, Ohio. Correspondence: H. William Schnaper (schnaper@northwestern.edu)

Received 4 June 2017; accepted 18 June 2017; advance online publication 30 August 2017. doi:10.1038/pr.2017.180
} 
Review | schnaper et al.

Table 1. Composition of the ASPN Therapeutics Development Committee

\begin{tabular}{ll}
\hline Participant representation & Number of members \\
\hline Pediatric nephrologists & Seven, including two ASPN officers ex-officio \\
Industry & Six different firms represented \\
NIDDK & One member \\
NICHD & One member \\
FDA & Three members, two of whom are pediatric nephrologists; one each in Center for Drug Evaluation \\
& of Research (CDER), Division of Cardiovascular and Renal Products; CDER, Division of Pediatrics \\
and Maternal Health; and Center for Devices and Radiological Health.
\end{tabular}

in academic institutions may discourage physicians from becoming site principal investigators for clinical trials that offer little local return for the time commitment. It is also important to consider issues related to liability, practicality, and ethics. In addition, patient enrollment may be limited by parental concerns related to study burdens, including missed school, painful procedures, time away from the parents' work, and varied parental attitudes toward clinical research in their children. In pediatric nephrology, these challenges include the varied rates at which chronic kidney disease progresses in children and the relative scarcity of validated and appropriate biomarkers for pediatric kidney disease studies. (10)

\section{AMERICAN SOCIETY OF PEDIATRIC NEPHROLOGY THERAPEUTICS DEVELOPMENT COMMITTEE}

In response to these concerns, in 2012 the American Society of Pediatric Nephrology (ASPN) created a Therapeutics Development Committee (TDC). It was felt that the issues described here have exceptional impact in pediatric nephrology. Beyond a few well-established programs, most pediatric nephrologists have not been in a position to participate in or contribute to the design of drug and device studies. Examples of consortia in other pediatric subspecialties that have addressed this problem include the large and longestablished Children's Oncology Group (COG) and the Childhood Arthritis and Rheumatology Research Alliance (CARRA). The intent of the TDC was to bring to the table as many stakeholders in pediatric nephrology therapeutics as possible, with the goal of addressing concerns that are specific to developing future treatments for pediatric kidney diseases. It was felt that, in a procedure-heavy but small subspecialty, a key issue in early-childhood application of compounds and devices is communication. Even if not all of the difficulties cited here can be overcome, ongoing interactions would better identify needs, develop ideas, and enhance and accelerate successful trials in pediatric nephrology.

Initially, the members of the Committee represented academic pediatric nephrologists, a patient advocacy group, members of industry, and representatives of the FDA and the National Institutes of Health (NIH). However, we quickly realized that the spectrum of potential contributors is much broader, and we have continued to add members as we have become aware of appropriate individuals. Table 1 lists the stakeholders that currently comprise the TDC.

\section{GOALS, ACTIVITIES, AND FINDINGS OF THE COMMITTEE}

The first goal of the TDC was to develop lines of communication among the disparate stakeholders. Conversations among pediatric nephrologists and representatives of industry and the FDA helped to orient these parties to the need for and limitations of pediatric studies. The FDA was extremely helpful in clarifying the regulatory considerations related to clearance and approval of new therapies. A key lesson learned through these discussions was that designing and planning clinical trials by one group in isolation, with minimal input from the perspective of other key stakeholders, was unlikely to be as successful as a joint effort. Without collaboration, a trial developed from industry might have unrealistic expectations for the volume and frequency of clinical samples or the frequency or intensity of visits. A trial developed in an academic center might have insufficient consideration of the types and quality of data needed to meet regulatory standards for FDA approval. Increased communication early in the process is clearly essential to mitigate such problems and support trials designed in a manner that could meet the data requirements of the FDA, be clinically translatable, and allow for successful study enrollment and completion. These conversations led to improved networking to facilitate and accelerate the design of further studies. The anticipated result is increased efficiency and the development of products ideally directed toward pediatric health needs.

The group began a project to develop a "road map" of the pathway for conducting drug and device studies. Our initial version of this road map, created by academic pediatric nephrologists with input from the FDA (Figure 1), shows the steps involved in developing and performing a study while providing expert resources for consultation if difficulties arise. An industry-based road map was also designed, to better understand the general process followed for companysponsored studies and implemented by a Research \& Development team (Figure 2). Both road maps are presented to illustrate the similarities and differences 

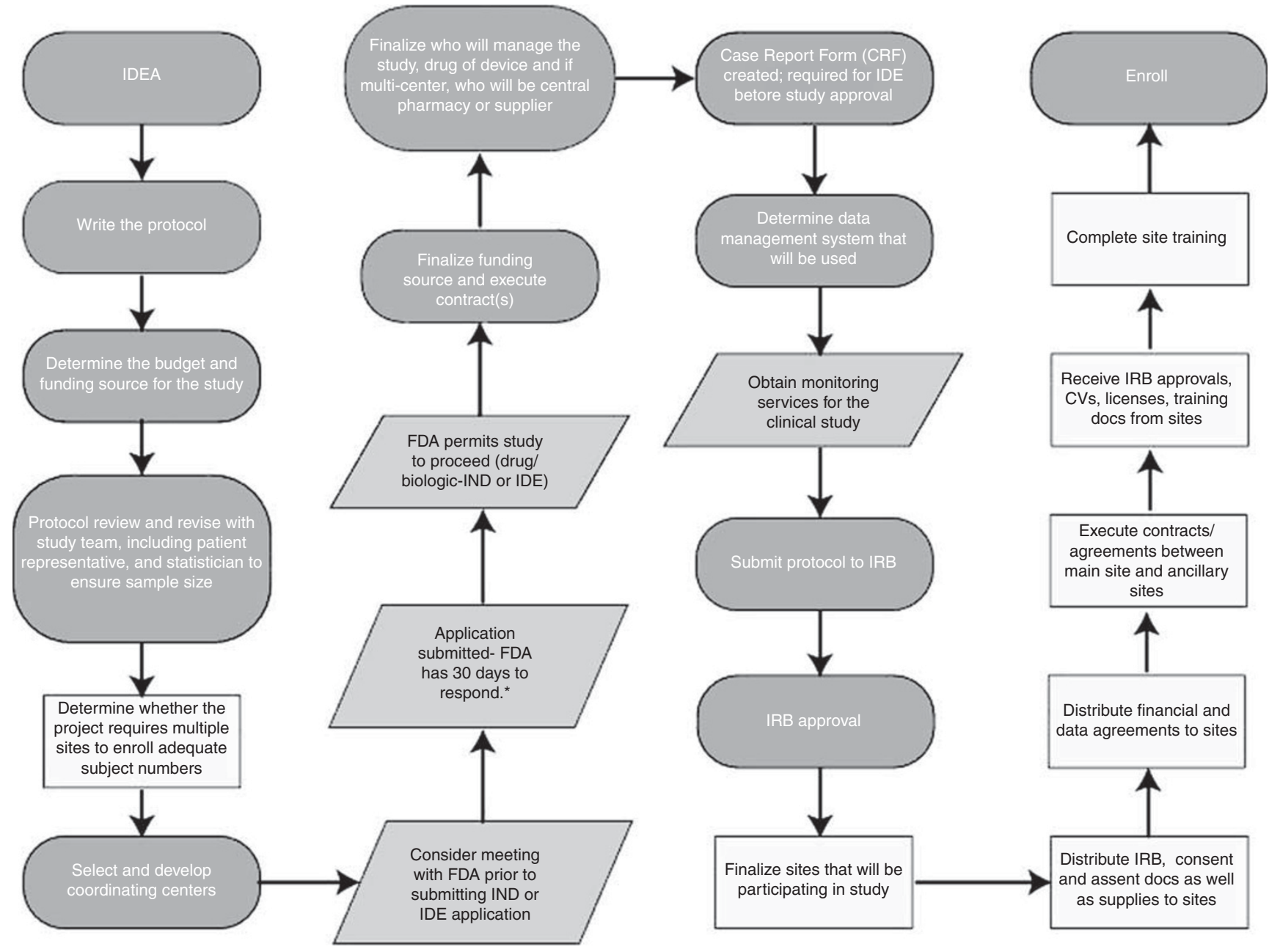

Figure 1. Academic approach to Clinical Trial Design. The sequential steps involved in clinical trial development and execution are detailed. Blue ovals represent internal planning steps, whereas gray rectangles represent multisite planning steps, and green rhomboids represent regulatory steps. Points of intersection between this diagram and those in Figure 2 are indicated by using bold for the critical words in the text. ${ }^{*} \mathrm{CDER}$ may request a change with or without putting a "hold" on the study; CDRH may approve or disapprove the study. A full color version of this figure is available at the Pediatric RESEARCH journal online, as well as online at the ASPN website (see text for details).

between the investigator-initiated and industry-based approaches. The steps can be categorized as involving internal planning, external organization of the participating research sites, and the actions required to obtain regulatory approval. Steps that are primarily related to each of these three areas are indicated by common shapes and colors. Although most of the essential steps are covered by the charts in both figures, the granularity of analysis for different stages of the process differs significantly. Figure 1 reflects the perspective of the academic investigator seeking to conduct a study on a product and emphasizes concept, whereas the industry approach shown in Figure 2 is likely self-funded and emphasizes the complex processes required to organize the study and ultimately move the product toward regulatory approval. We believe that there is clear value in both perspectives, with the differences not only being informative to the various stakeholders but also illuminating potential opportunities for synergy and the importance of maintaining an active dialogue to continue addressing differences in process and culture. Finally, there is growing consensus that patient involvement in research may increase patient enrollment in studies, the credibility and applicability of study results, and the translation of results into clinical practice. Inclusion of children and parents in this role is thus represented in both figures. Parents are typically included as representatives of young children, although an effort should be made to include adolescent patients in the decision-making process when appropriate. Lay public involvement will both enhance the conduct of the trial and increase subsequent acceptability of the product to the public (and therefore effective treatment).

The charts are posted on the public part of the ASPN website at http://www.aspneph.org/Clinical_Trial_Develop ment/ClinicalTrialsDevMain.cfm.

Pop-up windows are being developed for each step along the path of trial design and execution to indicate where people 


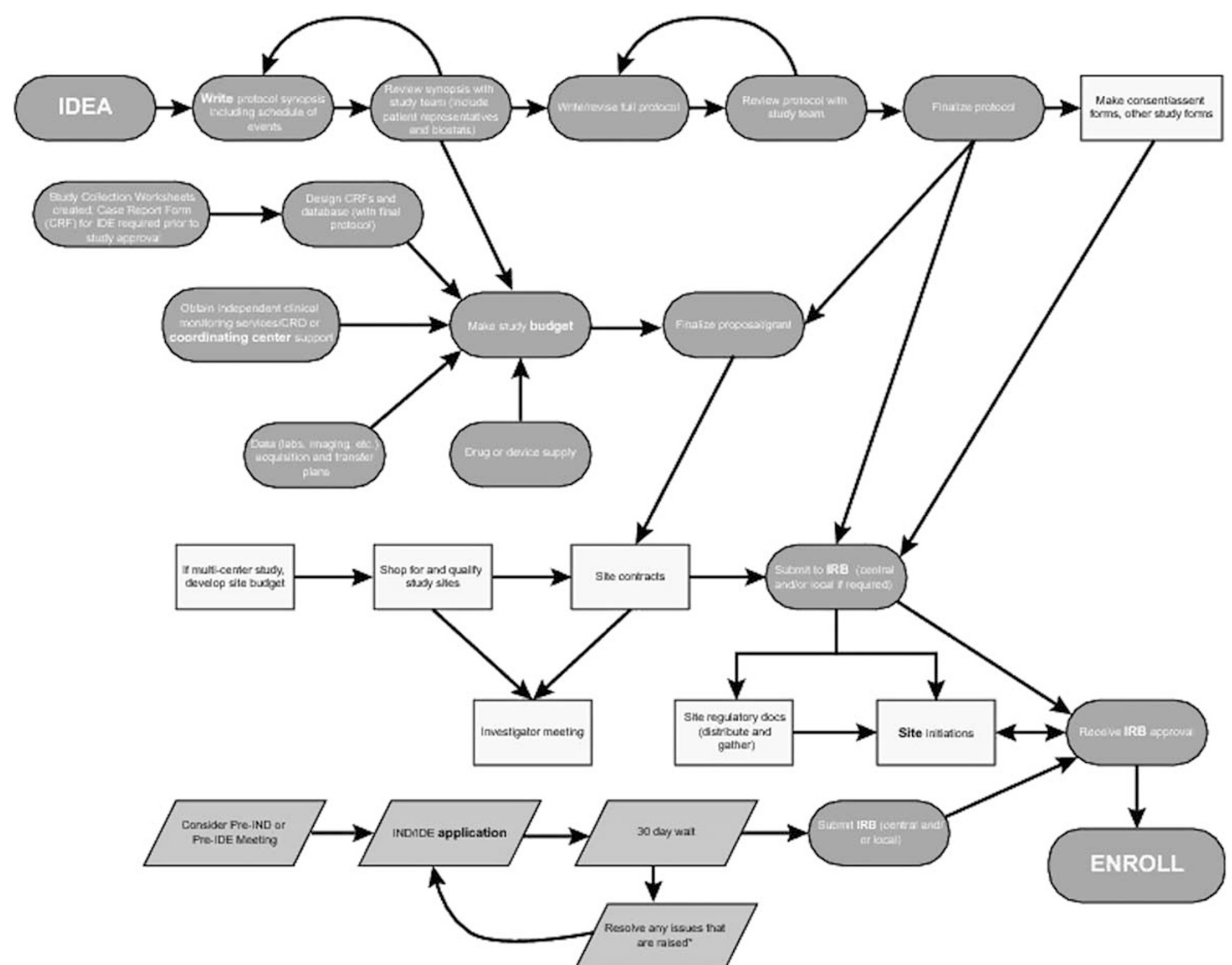

Figure 2. Industry approach to Clinical Trial Design. The sequential steps involved in clinical trial development and execution are detailed. Blue ovals represent internal planning steps, whereas gray rectangles represent multisite planning steps, and green rhomboids represent regulatory steps. Points of intersection between this diagram and those in Figure $\mathbf{1}$ are indicated by using bold for the critical words in the text. For additional information on the Industry approach to clinical trials, the reader is referred to the Common Protocol developed by TransCelerate, a consortium of biopharmaceutical companies: http://www.transceleratebiopharmainc.com/assets/common-protocol-template/. *CDER may request a change with or without putting a "hold" on the study; CDRH may approve or disapprove the study. A full color version of this figure is available at the Pediatric RESEARCH journal online, as well as online at the ASPN website (see text for details). 
Table 2. Functions carried out by the ASPN Therapeutics Development Committee

\begin{tabular}{ll}
\hline Activity & Participants \\
\hline $\begin{array}{l}\text { Identifying therapeutic needs } \\
\text { Evaluating treatments }\end{array}$ & All \\
$\begin{array}{l}\text { Identifying scientific needs } \\
\text { Ensuring that patient perspective is }\end{array}$ & Academic, NIDDK, NICHD \\
$\begin{array}{l}\text { included } \\
\text { Identifying PI partners and patient } \\
\text { populations }\end{array}$ & Foundations, PCORI \\
$\begin{array}{l}\text { Assuring adequacy of studies } \\
\text { Enhancing collaborative relationships } \\
\text { among stakeholders }\end{array}$ & Academic, industry \\
\hline ASPN, American Society of Pediatric Nephrology. &
\end{tabular}

or resources might be available to direct interested parties toward assistance they might need to complete each step. By maintaining the charts on the website, it will be possible to update them in real time, as needed.

\section{EXPANDED MISSION OF THE COMMITTEE}

The TDC was established originally to develop improved approaches for clinical trial design, but its members have realized that there is significant value in simply talking, exchanging viewpoints, and developing relationships that can be used to facilitate the conduct of future trials. Accordingly, the members plan to continue ongoing interactions semiannually at professional meetings and additionally by e-mail. As these discussions have progressed, we have recognized the need to expand the group. Table 2 shows the many contributions by different stakeholders to the committee's activities.

Alternative approaches to that of the TDC merit consideration. A single umbrella study group such as COG or CARRA did not develop originally in pediatric nephrology; instead, multiple clinical research groups formed with more narrow fields of interests. Perhaps this need for focused groups resulted from time (e.g., workforce issues) or geographic (e.g., wide dispersal of potential subjects) constraints. The ASPN, given its multiple functions as a professional society, does not desire to oversee and conduct clinical trials, instead choosing to serve as an "honest broker" facilitating interactions. We believe that a large umbrella group can facilitate clinical trials by expanding the roster of potential research sites and subjects, efficiently utilizing the small population of potential subjects and minimizing duplicated effort.

As such a program grows, it will be important to encourage inclusivity for participants in the design and conduct of studies as well as continuing to support conversation among representatives of various stakeholders presently embodied by the TDC. Ongoing inclusivity is an important consideration in facilitating clinical trials in a small academic community. Therefore, TDC will continue to seek members from among various stakeholders, adding to the list shown in the tables. In addition, coordination with other groups that have similar goals is essential. Representatives of several pediatric nephrology clinical trial groups participate in TDC, and TDC has representation in an ongoing Kidney Health Initiative (KHI) project that recently has been established as a collaboration between the American Society of Nephrology and FDA to identify and address roadblocks to clinical trials in pediatric nephrology.

\section{FUTURE PLANS}

As the activities of the TDC mature, it is our expectation that there will be increasing value derived for all participants by having an ongoing, robust forum for sharing viewpoints, concerns, changes in relevant rules or laws, and suggestions from all stakeholders involved in developing treatments for children with kidney disease. We hope that publication of this paper will increase awareness of the TDC and will encourage others to join this initiative. Ultimately, we anticipate that the enhanced relationships and trust derived from these ongoing interactions will greatly accelerate the development and approval of innovative future therapies for children with kidney disease.

\section{CONCLUSION}

The ASPN Therapeutics Development Committee was formed with the goals of (1) identifying the relevant stakeholders involved in the development, testing, and regulatory approval of new treatments for children with kidney disease and (2) developing more effective partnerships among all parties regarding strategies for successful clinical trial development and execution. Our work to date has highlighted the similarities and differences between the approaches of academic investigators and industry. It is hoped that continued maturation of these relationships and ongoing collaboration among the stakeholders will significantly accelerate the successful development and regulatory approval of new approaches to the care of children with renal disease.

\section{ACKNOWLEDGMENTS}

We appreciate the superb effort toward developing the figures contributed by Lisa Feurer. This article reflects the views of the authors and should not be construed to represent FDA's views or policies.

\section{STATEMENT OF FINANCIAL SUPPORT}

Supported in part by National Institutes of Health's National Center for Advancing Translational Sciences, Grants Number UL1TR001422 (H.W.S) and UL1TR001070 (W.E.S.).

Disclosure: C.G. is an employee of Relypsa Inc., a Vifor Pharma company. L. B.P. is an employee of Mallinckrodt Pharmaceuticals and owns stock in that company. The remaining authors declare no conflict of interest.

\section{REFERENCES}

1. Committee on Pediatric Studies Conducted Under the Best Pharmaceuticals for Children Act and the Pediatric Research Equity Act, Board on Health Sciences Policy, Institute of Medicine. In: Field MJ, Boat TF, eds. Safe and Effective Medicines for Children: Pediatric Studies Conducted Under the Best Pharmaceuticals for Children Act and the 


\section{Review | Schnaper et al.}

Pediatric Research Equity Act. Washington, DC: National Academies Press, 2012.

2. Shah SS, Hall M, Goodman DM, et al. Off-label drug use in hospitalized children. Arch Pediatr Adolesc Med 2007;161:282-90.

3. Sachs AN, Avant D, Lee CS, Rodriguez W, Murphy MD. Pediatric information in drug product labeling. JAMA 2012;307:1914-5.

4. Breslow LH. The Best Pharmaceuticals for Children Act of 2002: the rise of the voluntary incentive structure and congressional refusal to require pediatric testing. Harvard J Legis 2003;40:133-93.

5. Bourgeois FT, Hwang TJ. The Pediatric Research Equity Act Moves Into Adolescence. JAMA 2017;317:259-60.

6. Regulation (EC) No 1901/2006 of the European Parliament and of the Council of 12 December 2006.
7. Regulation (EC) No 1902/2006 of the European Parliament and of the council of 20 December 2006.

8. U.S. Department of Health and Human Services. Leveraging Existing Clinical Data for Extrapolation to Pediatric Uses of Medical Devices Guidance for Industry and Food and Drug Administration Staff. Rockville, MD: U.S. Department of Health and Human Services, Food and Drug Administration, Center For Devices and Radiological Health, Center for Biologics Evaluation and Research, 2016.

9. Frattarelli DA, Galinkin JL, Green TP, et al. Off-label use of drugs in children. Pediatrics 2014;133:563-7.

10. Schnaper HW, Furth SL, Yao LP. Defining new surrogate markers for CKD progression. Pediatr Nephrol 2015;30:193-8. 